\title{
The Changing Landscape of Alopecia Areata: An Introduction
}

\author{
George Han
}

Published online: June 23, 2017

(c) The Author(s) 2017. This article is an open access publication

\section{ABSTRACT}

Alopecia areata is an extremely common autoimmune condition affecting hair. Severe forms of alopecia areata exist, with existing treatments consisting of systemic immunosuppressants with numerous side effects. Recently, breakthroughs have been made in both understanding the pathogenesis of alopecia areata and the treatment thereof, which hold the promise of being able to target severe cases of alopecia areata with more efficacy and better tolerability. This article serves as an introduction to review papers from two of the leading researchers in the field of alopecia areata.

Keywords: Alopecia areata; JAK inhibitor; Immunosuppressant; Autoimmune; Hair loss

This is an editorial introduction to Wang ECE, Christiano AM. The changing landscape of alopecia areata: the translational landscape. Adv Ther. 2017 and Renert-Yuval Y, Guttman-Yassky E. The changing landscape of alopecia areata: the therapeutic paradigm.

G. Han $(\bowtie)$

Icahn School of Medicine at Mount Sinai, New York, NY, USA

e-mail: george.han@mountsinai.org
Although alopecia areata in its common presentation as patches of hair loss on the scalp is fairly innocuous and facile to treat, more severe forms of alopecia areata (AA) including totalis and universalis have long been very challenging conditions, with no standard of care for treatment. Treatment options for these severe forms of alopecia areata include immunosuppressants with numerous side effects and even when they are effective, a durable response is not common. Much of the difficulty underlying this lack of an effective treatment has been an incomplete understanding of the pathogenesis of AA. While the histologic characteristics of alopecia areata are well described, the causes of this pattern of inflammation have been elusive until recently.

In this issue, two of the pioneers of alopecia areata research, Dr. Angela Christiano and Dr. Emma Guttman-Yassky, have provided a comprehensive review of the current landscape of alopecia areata. In the first paper from Dr. Christiano's group [1], the immunopathogenesis of alopecia areata is explored, including the connections between in vivo, preclinical, and translational research studies. Genetic studies have provided a wealth of information about what inflammatory pathways underlie AA and when combined with a more refined understanding of the immune profile and cytokine activation in AA, our understanding of the disease process has improved greatly over recent years. Novel animal models, imaging modalities, and disease classification metrics are also 
presented. Finally, the rationale behind using various treatment modalities is discussed, providing an approach to understanding where they fit in to a treatment regimen. Accordingly, Dr. Guttman-Yassky's paper [2] takes a deeper dive into several newer treatment options for $\mathrm{AA}$, emphasizing the current evidence and data available for each medication and how they might fit into the clinical approach to AA. Using the treatment of psoriasis as a parallel paradigm in the treatment of a systemic inflammatory disease, the various cytokine pathways activated in AA in the context of drugs targeting them are summarized. Furthermore, specific differences between medications within the same class are presented, allowing for a more nuanced view of AA treatment options.

Together, these papers provide a timely review during a period where our understanding and treatment approach to AA is truly undergoing a revolution. Whereas we previously had only a very basic understanding of the pathogenesis of AA, we now have a wealth of information about mechanisms underlying AA which may lead to further discoveries and treatment approaches in coming years. Concordantly, novel treatments for AA are emerging that have the potential to transform patients' lives who have had to deal with the severe consequences of widespread AA. At this confluence of research and therapeutics, there is new hope in our approach to and treatment of alopecia areata.

Open Access. This article is distributed under the terms of the Creative Commons Attribution-NonCommercial 4.0 International License (http://creativecommons.org/licenses/ by-nc/4.0/), which permits any noncommercial use, distribution, and reproduction in any medium, provided you give appropriate credit to the original author(s) and the source, provide a link to the Creative Commons license, and indicate if changes were made.

\section{REFERENCES}

1. Wang ECE, Christiano AM. The changing landscape of alopecia areata: the translational landscape. Adv Ther. 2017. doi:10.1007/s12325-017-0540-9.

2. Renert-Yuval Y, Guttman-Yassky E. The changing landscape of alopecia areata: the therapeutic paradigm. Adv Ther. 2017. doi:10.1007/s12325-017-05427. 